\title{
Pacific
}

Journal of

Mathematics

\section{THE SIEGEL-WEIL FORMULA FOR UNITARY GROUPS}

SHUNSUKE YAMANA 


\title{
THE SIEGEL-WEIL FORMULA FOR UNITARY GROUPS
}

\author{
SHUNSUKE YAMANA
}

\section{We extend the Siegel-Weil formula for unitary groups of hermitian forms over a skew field with involution of the second kind.}

\section{Introduction}

The Siegel-Weil formula is an identity between an Eisenstein series and an integral of a theta function. After Weil [1965] proved such an identity when both sides of the identity are absolutely convergent, Kudla and Rallis [1988a; 1988b; 1994] extended it for symplectic groups beyond the range of absolute convergence. Their results were extended to almost all classical groups by several authors, of which we mention the following sample: [Tan 1998; Ichino 2004; 2007; Gan and Takeda 2011; Yamana 2011; 2013; Gan 2000]. In this paper we discuss the last case that has to be considered in the theory of classical dual pairs over a number field, namely, unitary groups of hermitian forms over a skew field with involution of the second kind.

Let $E / F$ be a quadratic extension of number fields and $D$ a division algebra with center $E$, of dimension $\delta^{2}$ over $E$ and provided with an antiautomorphism $\rho$ of order two under which $F$ is the fixed subfield of $E$. Let $\mathbb{A}$ and $\mathbb{A}_{E}$ be the rings of adeles of $F$ and $E$, respectively. Let $W$ be a left $D$-vector space of dimension $2 n$ with a nondegenerate skew hermitian form that has a complete polarization, and $V$ a right $D$-vector space of dimension $m$ with a nondegenerate hermitian form. Let $G$ and $H$ be the unitary groups of $\mathscr{W}$ and $V$, respectively.

Let $\alpha_{E}$ denote the standard norm of $\mathbb{A}_{E}^{\times}$. A character of $\mathbb{A}_{E}^{\times}$is called principal if it is a complex power of $\alpha_{E}$. We denote by $P$ the maximal parabolic subgroup of $G$ that stabilizes a maximal isotropic subspace of $W$. Note that $P$ has a Levi decomposition $P=M N$ with $M \simeq \mathrm{GL}_{n}(D)$. For any unitary character $\chi$ of $\mathbb{A}_{E}^{\times} / E^{\times}$ and for any $s \in \mathbb{C}$, we consider the representation $I(s, \chi)=\operatorname{Ind}_{P(\mathbb{A})}^{G(\mathbb{A})} \chi \alpha_{E}^{s}$ induced from the character $m \mapsto \chi(v(m)) \alpha_{E}(v(m))^{s}$, where $v$ is the reduced norm viewed

The author is supported by JSPS Grant-in-Aid for Research Activity Start-up 24840033. This work is partially supported by the JSPS Institutional Program for Young Researcher Overseas Visits "Promoting international young researchers in mathematics and mathematical sciences led by OCAMI".

MSC2010: primary 11F27; secondary 11F70.

Keywords: Eisenstein series, Siegel-Weil formula, degenerate principal series, doubling method. 
as a character of the algebraic group $\mathrm{GL}_{n}(D)$ and the induction is normalized so that $I(s, \chi)$ is naturally unitarizable when $s$ is pure imaginary. For any holomorphic section $f^{(s)}$ of $I(s, \chi)$, the Eisenstein series

$$
E\left(g ; f^{(s)}\right)=\sum_{\gamma \in P(F) \backslash G(F)} f^{(s)}(\gamma g)
$$

is absolutely convergent for $\Re s>\delta n / 2$ and has a meromorphic continuation to the whole $s$-plane. We denote by $\chi^{0}$ the restriction of $\chi$ to $\mathbb{A}^{\times}$, by $\rho(\chi)$ the character defined by $\rho(\chi)(x)=\chi\left(x^{\rho}\right)$ for $x \in \mathbb{A}_{E}^{\times}$, and by $\epsilon_{E / F}$ the quadratic character of $\mathbb{A}^{\times} / F^{\times}$associated to the extension $E / F$. The following theorem was proven in [Tan 1999] when $\delta=1$.

Theorem 1. Let $f^{(s)}$ be a holomorphic section of $I(s, \chi)$.

(1) If $\chi \rho(\chi)$ is not principal, then $E\left(g ; f^{(s)}\right)$ is entire.

(2) If $\chi=\rho(\chi)^{-1}$, then the poles of $E\left(g ; f^{(s)}\right)$ in $\Re s>-\frac{1}{2}$ are at most simple and can only occur in the set

$$
\left\{\frac{\delta(n-j)}{2} \mid j \in \mathbb{Z}, 0 \leq j<n, \chi^{0}=\epsilon_{E / F}^{\delta j}\right\} .
$$

Fix a nontrivial additive character $\psi$ of $\mathbb{A} / F$ and a character $\chi_{V}$ of $\mathbb{A}_{E}^{\times} / E^{\times}$such that $\chi_{V}^{0}=\epsilon_{E / F}^{\delta m}$. The group $G(\mathbb{A}) \times H(\mathbb{A})$ acts on the Schwartz space $\mathscr{S}\left(V^{n}(\mathbb{A})\right)$ of $V^{n}(\mathbb{A})$ via the Weil representation $\omega_{\psi, V, \chi_{V}}$. Let $S\left(V^{n}(\mathbb{A})\right)$ be the subspace of $\mathscr{S}\left(V^{n}(\mathbb{A})\right)$ consisting of functions that correspond to polynomials in the Fock model at every archimedean place of $F$.

The theta function associated to $\Phi \in S\left(V^{n}(\mathbb{A})\right)$ is defined by

$$
\Theta(g, h ; \Phi)=\sum_{x \in V^{n}(F)} \omega_{\psi, V, \chi_{V}}(g) \Phi\left(h^{-1} x\right)
$$

for $g \in G(\mathbb{A})$ and $h \in H(\mathbb{A})$. By Weil's criterion [1965], the integral

$$
I(g ; \Phi)=\int_{H(F) \backslash H(\mathbb{A})} \Theta(g, h ; \Phi) d h
$$

is absolutely convergent for all $\Phi$ either if $r=0$ or if $m-r>n$, where $r$ is the dimension of a maximal totally isotropic subspace of $V(F)$. When $m \leq n$ and $r>0$, the integral diverges in general, but extends uniquely to a $G(\mathbb{A})$-intertwining, $H(\mathbb{A})$-invariant map on $S\left(V^{n}(\mathbb{A})\right)$ in light of the regularization introduced by Kudla and Rallis [1994].

For $\Phi \in S\left(V^{n}(\mathbb{A})\right)$ we define a section $f_{\Phi}^{(s)}$ of $I\left(s, \chi_{V}\right)$ by

$$
f_{\Phi}^{(s)}(g)=|a(g)|^{s-s_{0}} \omega_{\psi, V, \chi_{V}}(g) \Phi(0),
$$


where $g \in G(\mathbb{A}), s_{0}=\delta(m-n) / 2$ and the quantity $|a(g)|$ is defined in the notation section below.

Theorem 2. If $m \leq n$ or if $m-r>n$, then for all $\Phi \in S\left(V^{n}(\mathbb{A})\right)$ the series $E\left(g ; f_{\Phi}^{(s)}\right)$ is holomorphic at $s=s_{0}$ and

$$
\left.E\left(g ; f_{\Phi}^{(s)}\right)\right|_{s=s_{0}}=\varkappa I(g ; \Phi),
$$

where

$$
\varkappa= \begin{cases}2 & \text { if } m \leq n, \\ 1 & \text { if } m-r>n .\end{cases}
$$

Theorem 2 was proven in [Weil 1965] if $m>2 n$, and in [Tan 1998; Ichino 2004; 2007; Yamana 2011] if $\delta=1$. The proof requires only slight technical modifications once all of the necessary local facts have been established. The group $G\left(F_{v}\right)$ is isomorphic to the quasisplit unitary group $U(\delta n, \delta n)$ or an inner form of $\mathrm{GL}_{2 \delta n}\left(F_{v}\right)$, depending on whether $v$ remains prime or splits in $E$. The former case has already been discussed in [Kudla and Sweet 1997; Ichino 2007; Lee and Zhu 1998], and the latter case is discussed in Section 1. Coupled with the doubling method, the Siegel-Weil formula relates the theory of theta liftings to the theory of automorphic $L$-functions. We study the doubling zeta integral for inner forms of general linear groups in the Appendix.

\section{Notation}

Let $(D, E, F, \rho)$ be as in the introduction. The restriction of $\rho$ to $E$, which we denote also by $\rho$, is the nontrivial automorphism of $E$ over $F$. For a matrix $x$ with entries in $D$, let $x^{*}={ }^{t} x^{\rho}$ be the conjugate transpose of $x$. If $x$ is a square matrix, then $v(x)$ and $\tau(x)$ stand for its reduced norm and reduced trace to $E$.

Fix a natural number $n$ and put $n^{\prime}=\delta n$. Let $\mathcal{W}=D^{2 n}$ be a left $D$-vector space with the skew hermitian form

$$
\langle x, y\rangle=x J y^{*}, \quad J=\left(\begin{array}{cc}
0 & \mathbf{1}_{n} \\
-\mathbf{1}_{n} & 0
\end{array}\right)
$$

for $x, y \in \mathcal{W}$. Let $V$ be a right $D$-vector space of dimension $m$ equipped with a nondegenerate hermitian form (, ). We denote by $G$ (resp. $H$ ) the group of all $D$-linear transformations of $\mathcal{W}$ (resp. $V$ ) that leave $\langle$,$\rangle (resp. (, )) invariant. Put$ $s_{0}=\delta(m-n) / 2$.

We write $P$ for the stabilizer in $G$ of the maximal isotropic subspace of $\mathcal{W}$ defined by the vanishing of all but the last $n$ coordinates. Let

$$
\operatorname{Her}_{n}=\left\{x \in \mathrm{M}_{n}(D) \mid x^{*}=x\right\}
$$


be the $F$-subvariety of $n \times n$ hermitian matrices. The group $G$ has a maximal parabolic subgroup $P=M N$ given by

$$
\begin{aligned}
& M=\left\{m(a)=\left(\begin{array}{cc}
a & 0 \\
0 & \left(a^{-1}\right)^{*}
\end{array}\right) \mid a \in \mathrm{GL}_{n}(D)\right\}, \\
& N=\left\{n(b)=\left(\begin{array}{cc}
\mathbf{1}_{n} & b \\
0 & \mathbf{1}_{n}
\end{array}\right) \mid b \in \operatorname{Her}_{n}\right\} .
\end{aligned}
$$

Let $K$ be the standard maximal compact subgroup of $G(\mathbb{A})$. For any character $\chi$ of $\mathbb{A}_{E}^{\times} / E^{\times}$, the representation $I(s, \chi)=I_{n^{\prime}}(s, \chi)$ is realized on the space of right $K$-finite functions $f^{(s)}: G(\mathbb{A}) \rightarrow \mathbb{C}$ satisfying

$$
f^{(s)}(m(a) n(b) g)=\chi(v(a)) \alpha_{E}(v(a))^{s+n^{\prime} / 2} f^{(s)}(g)
$$

for all $a \in \mathrm{GL}_{n}(D(\mathbb{A})), b \in \operatorname{Her}_{n}(\mathbb{A})$ and $g \in G(\mathbb{A})$. We define $|a(g)|$ by writing $g=p k \in G(\mathbb{A})$ with $p=m(a) n(b) \in P(\mathbb{A})$ and $k \in K$, and taking $|a(g)|=\alpha_{E}(v(a))$.

\section{Degenerate principal series representations}

For each place $v$ of $F$, let $F_{v}$ be the $v$-completion of $F$ and set $E_{v}=E \otimes_{F} F_{v}$ and $D_{v}=D \otimes_{F} F_{v}$. A division algebra $D$ with center $E$ admits an involution of the second kind if and only if $D_{v}$ is isomorphic to $\mathrm{M}_{\delta}\left(E_{v}\right)$ whenever $v$ remains prime in $E$, and $D_{v}$ is isomorphic to a direct sum of mutually opposite simple algebras whose centers are $F_{v}$ whenever $v$ splits in $E$ (see [Scharlau 1985, Theorem 10.2.4]).

In the local setting we will depart slightly from our previous notation. Fix a place $v$ of $F$ and suppress it from the notation. Thus $E$ is a quadratic étale algebra over the local field $F, D$ an algebra whose center is $E, \rho$ an involution of $D$ whose restriction to $E$ is the nontrivial automorphism of $E$ over $F, V$ a free right $D$-module of rank $m$, and (, ) $: V \times V \rightarrow D$ an $F$-bilinear map satisfying the following conditions:

- for $a, b \in D$ and $x, y \in V$,

$$
(x, y)^{\rho}=(y, x), \quad(x a, y b)=a^{\rho}(x, y) b ;
$$

- $(x, V)=0$ implies that $x=0$.

Let $H$ be the unitary group of $V$. Let $G=\left\{g \in \mathrm{GL}_{2 n}(D) \mid g J g^{*}=J\right\}$. For any quasicharacter $\chi$ of $E^{\times}$, let $I(s, \chi)$ be the analogous local induced representation of $G$. By Morita context, it is enough to consider the case where the triple $(D, E, \rho)$ belongs to the following two types:

- $D=E$ is a quadratic extension of $F$ and $\rho$ generates $\operatorname{Gal}(E / F)$;

- $D=\boldsymbol{D} \oplus \boldsymbol{D}^{\text {op }}, E=F \oplus F$ and $(x, y)^{\rho}=(y, x)$, where $\boldsymbol{D}$ is a division algebra central over $F$ and $\boldsymbol{D}^{\text {op }}$ is its opposite algebra. 
The rank of $D$ as a module over $E$ is a square of a natural number that will be denoted by $\delta$. Note that $n^{\prime}=\delta n$ remains intact after the change in notation.

We fix a nontrivial additive character $\psi$ of $F$ and a character $\chi_{V}$ of $E^{\times}$that satisfies $\chi_{V}^{0}=\epsilon_{E / F}^{\delta m}$. Then $G \times H$ acts on the Schwartz space $\mathscr{S}\left(V^{n}\right)$ via the Weil representation $\omega_{\psi, V, \chi_{V}}$. Note that it depends on the data $\psi,\left(\right.$, ) and $\chi_{V}$ (compare [Kudla 1994]). When $F$ is a $p$-adic field, put $S\left(V^{n}\right)=\mathscr{S}\left(V^{n}\right)$. When $F=\mathbb{R}$ or $\mathbb{C}$, let $\mathfrak{g}$ be the complexified Lie algebra of $G$ and $S\left(V^{n}\right)$ the subspace of $\mathscr{S}\left(V^{n}\right)$ that corresponds to the space of polynomials in the Fock model of $\omega_{\psi, V, \chi_{V}}$. In the archimedean case we only consider admissible representations of the pair $(\mathfrak{g}, K)$, although we will allow ourselves to speak of a representation of the group $G$. We write $R\left(V, \chi_{V}\right)=R_{n^{\prime}}\left(V, \chi_{V}\right)$ for the image of the intertwining map

$$
S\left(V^{n}\right) \rightarrow I\left(s_{0}, \chi_{V}\right), \quad \Phi \mapsto f_{\Phi}^{\left(s_{0}\right)}(g)=\omega_{\psi, V, \chi_{V}}(g) \Phi(0) .
$$

We extend $f_{\Phi}^{\left(s_{0}\right)}$ to the standard section $f_{\Phi}^{(s)}$ of $I\left(s, \chi_{V}\right)$.

We discuss the case $E=F \oplus F$. Put

$$
e_{1}=(1,0), \quad e_{2}=(0,1), \quad V_{1}=V e_{1}, \quad V_{2}=V e_{2} .
$$

We regard $V_{1}$ as a right $\boldsymbol{D}$-module and $V_{2}$ as both a right $\boldsymbol{D}^{\mathrm{op}}$-module and a left $\boldsymbol{D}$-module. Since $\left(V_{i}, V_{i}\right)=0$ for $i=1,2$, the spaces $V_{1}$ and $V_{2}$ are paired nondegenerately against each other by $($, , , and so an antiisomorphism

$$
\jmath: \operatorname{End}\left(V_{1}, \boldsymbol{D}\right) \rightarrow \operatorname{End}\left(V_{2}, \boldsymbol{D}^{\text {op }}\right)
$$

is defined by

$$
(a x, y)=(x, J(a) y), \quad a \in \operatorname{End}\left(V_{1}, \boldsymbol{D}\right), x \in V_{1}, y \in V_{2} .
$$

We obtain

$$
H=\left\{\left(a, J(a)^{-1}\right) \in \mathrm{GL}\left(V_{1}, \boldsymbol{D}\right) \times \mathrm{GL}\left(V_{2}, \boldsymbol{D}^{\mathrm{op}}\right) \mid a \in \mathrm{GL}\left(V_{1}, \boldsymbol{D}\right)\right\} .
$$

Thus projection onto the first or second factor induces an isomorphism of $H$ onto $\operatorname{GL}\left(V_{1}, \boldsymbol{D}\right)$ or $\operatorname{GL}\left(V_{2}, \boldsymbol{D}^{\mathrm{op}}\right)$, respectively. For any nonnegative integer $j$ we write $G_{j}^{\prime}=\mathrm{GL}_{j}(\boldsymbol{D})$. Observe that

$$
G=\left\{\left(g, J^{-1} g^{-1} J\right) \mid g \in G_{2 n}^{\prime}\right\} .
$$

Through projection onto the first factor, we identify $H$ with $G_{m}^{\prime}, G$ with $G_{2 n}^{\prime}$, and $P=M N$ with

$$
M=\left\{\left(\begin{array}{ll}
a & 0 \\
0 & d
\end{array}\right) \mid a, d \in \mathrm{GL}_{n}(\boldsymbol{D})\right\}, \quad N=\left\{\left(\begin{array}{cc}
\mathbf{1}_{n} & b \\
0 & \mathbf{1}_{n}
\end{array}\right) \mid b \in \mathrm{M}_{n}(\boldsymbol{D})\right\} .
$$


We write $v=v_{j}$ for the reduced norm of $\mathbf{M}_{j}(\boldsymbol{D})$ and $\tau$ for the reduced trace of $\mathrm{M}_{j}(\boldsymbol{D})$. Let $\alpha_{F}(x)=|x|_{F}$ denote the normalized absolute value of $x \in F^{\times}$. When we write $\chi=\left(\chi_{1}, \chi_{2}\right)$, the representation $I(s, \chi)$ is translated to

$$
I(s, \chi)=\operatorname{Ind}_{P}^{G_{2 n}^{\prime}}\left(\left(\chi_{1} \alpha_{F}^{s}\right) \circ v_{n} \otimes\left(\chi_{2} \alpha_{F}^{s}\right)^{-1} \circ v_{n}\right) .
$$

If $E=F \oplus F$, then since $\chi_{V}$ is of the form $\left(\mu, \mu^{-1}\right)$, we may assume that $\chi_{V}=1$ by twisting, and we write $I(s)=I(s, 1)$ and $R(V)=R(V, 1)$. The Weil representation $\omega_{j, k}$ of the dual pair $\left(G_{j}^{\prime}, G_{k}^{\prime}\right)$ can be taken to be the action on $\mathscr{S}\left(\mathrm{M}_{k, j}(\boldsymbol{D})\right)$ given by

$$
\omega_{j, k}(a, b) \phi(x)=\alpha_{F}\left(v_{j}(a)\right)^{\delta k / 2} \alpha_{F}\left(v_{k}(b)\right)^{-\delta j / 2} \phi\left(b^{-1} x a\right)
$$

for $a \in G_{j}^{\prime}$ and $b \in G_{k}^{\prime}$. Note that the integral

$$
\left(\phi, \phi^{\prime}\right)=\int_{\mathrm{M}_{k, j}(\boldsymbol{D})} \phi(u) \overline{\phi^{\prime}(u)} d u, \quad \phi, \phi^{\prime} \in \mathscr{S}\left(\mathrm{M}_{k, j}(\boldsymbol{D})\right)
$$

defines a $G_{j}^{\prime} \times G_{k}^{\prime}$ invariant positive definite hermitian form on $\omega_{j, k}$. The two models of the Weil representation $\omega_{2 n, m} \simeq \omega_{\psi, V, 1}$ are related by the partial Fourier transform

$$
\mathscr{F} \phi(x, y)=\int_{\mathrm{M}_{m, n}(\boldsymbol{D})} \phi((x, z)) \psi\left(-\tau\left(z^{t} y\right)\right) d z
$$

for $x \in \mathrm{M}_{m, n}(\boldsymbol{D})$ and $y \in \mathrm{M}_{m, n}\left(\boldsymbol{D}^{\mathrm{op}}\right)$. In the $p$-adic case we write $\mathcal{O}$ for the maximal compact subring of $\boldsymbol{D}$ and put $K_{n}=\mathrm{GL}_{n}(\mathcal{O})$. In the archimedean case we set

$$
K_{n}=\left\{g \in G_{n}^{\prime} \mid{ }^{t} \bar{g} g=\mathbf{1}_{n}\right\},
$$

denoting the conjugate transpose of $x \in \mathrm{M}_{n}(\boldsymbol{D})$ by ${ }^{t} \bar{x}$, where ${ }^{-}$denotes the complex conjugate or the quaternion conjugate. We denote by $f_{0}^{(s)}$ a unique section of $I(s)$ that is identically 1 on $K_{2 n}$.

Lemma 1.1. If $E=F \oplus F$, then $R(V)$ contains $f_{0}^{\left(s_{0}\right)}$.

Proof. In the $p$-adic case, we let $\phi_{j, k}$ be the characteristic functions of $\mathrm{M}_{j, k}(\mathcal{O})$. In the archimedean case we let

$$
\phi_{j, k}(x)=e^{-\pi \operatorname{Tr}_{F / \mathbb{R}}\left(\tau\left(t_{\bar{x}} x\right)\right)},
$$

assuming that $\psi(\cdot)=e^{2 \pi \sqrt{-1} \operatorname{Tr}_{F / \mathbb{R}}(\cdot)}$. Put $\Phi=\mathscr{F} \phi_{2 n, m}$. Then $f_{\Phi}^{\left(s_{0}\right)}$ is nonzero and right invariant under $K_{2 n}$.

The local intertwining operator is defined analogously by

$$
M(s, \chi) f^{(s)}(g)=\int_{\operatorname{Her}_{n}(F)} f^{(s)}(\operatorname{Jn}(b) g) d b .
$$


We define holomorphic sections and standard sections similarly. We write $\chi^{0}$ for the restriction of $\chi$ to $F^{\times}$. Put

$$
\begin{aligned}
& a(s, \chi)=a_{n^{\prime}}(s, \chi)=\prod_{j=1}^{n^{\prime}} L\left(2 s-j+1, \chi^{0} \cdot \epsilon_{E / F}^{n^{\prime}+j}\right), \\
& b(s, \chi)=b_{n^{\prime}}(s, \chi)=\prod_{j=1}^{n^{\prime}} L\left(2 s+j, \chi^{0} \cdot \epsilon_{E / F}^{n^{\prime}+j}\right) .
\end{aligned}
$$

A normalized intertwining operator $M^{*}(s, \chi)$ is defined by setting

$$
M^{*}(s, \chi)=a(s, \chi)^{-1} M(s, \chi) .
$$

Lemma 1.2. The operator $M^{*}(s, \chi)$ is entire.

Proof. When $E / F$ is a quadratic extension of $p$-adic fields, Lemma 1.2 is proven in Proposition 3.2 of [Kudla and Sweet 1997]. The proof is completely analogous when $E / F=\mathbb{C} / \mathbb{R}$. Note that Proposition 3 A.6 of the same work applies also to this case by a global consideration, namely, by applying (24) of [Lapid and Rallis 2005] with base field $\mathbb{Q}$ and $S=\{\infty\}$.

We suppose that $E=F \oplus F$. For $\phi \in \mathscr{S}\left(\mathrm{M}_{n}(\boldsymbol{D})\right)$ we define a section $f_{\phi}^{(s)}$ of $I(s, \chi)$ by requiring that $\operatorname{supp}\left(f_{\phi}^{(s)}\right) \subset P J N$ and $f_{\phi}^{(s)}(g)=\phi(b)$ if $g=J n(b)$ for $b \in \operatorname{Her}_{n}(F)$. As explained in [Piatetski-Shapiro and Rallis 1987b; Kudla and Sweet 1997], all we have to do is to show that the ratio $a(s, \chi)^{-1} M(s, \chi) f_{\phi}^{(s)}(J)$ is entire. One can easily observe that

$$
M(s, \chi) f_{\phi}^{(s)}(J)=Z^{G J}\left(2 s-\frac{n^{\prime}}{2}, \phi, \chi^{0} \circ v_{n}\right),
$$

where the right-hand side is the zeta integral studied in [Weil 1974; Godement and Jacquet 1972] (see the Appendix). Our claim follows at once, as the GodementJacquet $L$-factor

$$
L^{G J}\left(2 s-\frac{n^{\prime}-1}{2}, \chi^{0} \circ v_{n}\right)
$$

divided by the factor $a(s, \chi)$ is entire.

For $\beta \in \operatorname{Her}_{n}(F)$, let $\psi_{\beta}$ be the character of $N$ defined by $\psi_{\beta}(n(b))=\psi(\tau(\beta b))$. Notice that $\tau(\beta b) \in F$. The Fourier transform of a Schwartz function $f \in \mathscr{S}(N)$ is defined by

$$
\hat{f}(\beta)=\int_{N} f(u) \psi_{\beta}(u) d u .
$$


For each integer $j \leq n^{\prime}$, we define the subvariety $\operatorname{Her}_{n}^{j}$ of $\operatorname{Her}_{n}(F)$ by

$$
\begin{array}{ll}
(E \not 7 F \oplus F) & \operatorname{Her}_{n}^{j}=\left\{\beta \in \mathbf{M}_{n}(E) \mid{ }^{t} \beta^{\rho}=\beta, \operatorname{rank}_{E} \beta \leq j\right\}, \\
(E=F \oplus F) & \operatorname{Her}_{n}^{j}=\left\{\left(\beta,{ }^{t} \beta\right) \in \mathbf{M}_{n}(\boldsymbol{D}) \oplus \mathbf{M}_{n}\left(D^{\text {op }}\right) \mid \delta\left(\operatorname{rank}_{\boldsymbol{D}} \beta\right) \leq j\right\} .
\end{array}
$$

Definition 1.3. We say that a representation $\pi$ of $G$ has rank at most $j$ if $f \in \mathscr{S}(N)$ acts by zero on $\pi$ whenever $\hat{f}$ vanishes on $\operatorname{Her}_{n}^{j}$. We say that $\pi$ is of rank $j$ if in addition $j$ is a multiple of $\delta$ and $\pi$ does not have rank less than $j$.

For any $H$-module $\pi$, we write $\pi_{H}$ for the maximal quotient of $\pi$ on which $H$ acts trivially. Let $\mathscr{H}_{r}$ be a split hermitian space of dimension $2 r$, that is, $\mathscr{H}_{r}$ has a $D$-basis consisting of $2 r$ elements $e_{i}, f_{i}$ such that

$$
\left(e_{i}, e_{j}\right)=\left(f_{i}, f_{j}\right)=0, \quad\left(e_{i}, f_{j}\right)=\delta_{i j} .
$$

Proposition 1.4. Assume that $m \leq n$. Let $U=V \oplus \mathscr{H}_{n-m}$.

(1) $R\left(V, \chi_{V}\right)$ is irreducible and unitarizable.

(2) $R\left(V, \chi_{V}\right)$ is isomorphic to $S\left(V^{n}\right)_{H}$.

(3) If $E / F$ is a quadratic extension of p-adic fields, then $R\left(V, \chi_{V}\right)$ is of rank $m$.

(4) $R\left(U, \chi_{V}\right)$ has a unique irreducible quotient that is isomorphic to $R\left(V, \chi_{V}\right)$.

(5) $M^{*}\left(-s_{0}, \chi_{V}\right)$ maps $R\left(U, \chi_{V}\right)$ onto $R\left(V, \chi_{V}\right)$.

(6) $b\left(s, \chi_{V}\right) M^{*}\left(s, \chi_{V}\right) f_{\Phi}^{(s)}$ is holomorphic at $s=s_{0}$ for every $\Phi \in S\left(V^{n}\right)$.

Proof. When $D=E$, these results are known (see [Li 1989; Mœglin et al. 1987; Kudla and Sweet 1997; Lee and Zhu 1998; Yamana 2011]). We may suppose that $E=F \oplus F$ and $\delta>1$.

For $0 \leq i \leq k$, let $P_{i}^{k}=M_{i}^{k} N_{i}^{k}$ be the maximal parabolic subgroup of $G_{k}^{\prime}$ given by

$$
P_{i}^{k}=\left\{\left(\begin{array}{ll}
a & b \\
0 & d
\end{array}\right) \in G_{k}^{\prime} \mid a \in G_{k-i}^{\prime}, b \in \mathrm{M}_{k-i, i}(\boldsymbol{D}), d \in G_{i}^{\prime}\right\},
$$

$\bar{P}_{i}^{k}$ its opposite parabolic subgroup, and $r_{i}$ the representation of $G_{i}^{\prime} \times G_{i}^{\prime}$ on $\mathscr{S}\left(G_{i}^{\prime}\right)$ given by

$$
r_{i}\left(g_{1}, g_{2}\right) \phi(g)=\phi\left(g_{2}^{-1} g g_{1}\right), \quad\left(\phi \in \mathscr{S}\left(G_{i}^{\prime}\right), g, g_{1}, g_{2} \in G_{i}^{\prime}\right) .
$$

In the archimedean case the representation $I(s)$ is studied extensively in [Lee 2007; Sahi 1995; Zhang 1995]. From their results we know the module structure of $I\left(s_{0}\right)$ and the set of $K$-types of each of its irreducible constituents, which combined with the technique explained in [Kudla and Rallis 1990a] prove (1), (2). We consider the nonarchimedean case. By Lemma 3.III.2 of [Mœglin et al. 1987], the representation $\omega_{2 n, m}$ has a filtration

$$
0 \subset S_{m} \subset \cdots \subset S_{1} \subset S_{0}=\omega_{2 n, m}
$$


with successive quotients

$$
S_{i} / S_{i+1} \simeq \operatorname{Ind}_{P_{i}^{2 n} \times \bar{P}_{i}^{m}}^{G_{2}^{\prime} \times G_{i}^{\prime}} \mu_{i},
$$

where $\mu_{i}$ is the representation of $P_{i}^{2 n} \times \bar{P}_{i}^{m}$ on $\mathscr{S}\left(G_{i}^{\prime}\right)$ given by

$$
\mu_{i}\left(p, p^{\prime}\right) \phi=\alpha_{F}\left(v(a)^{m-i} v\left(a^{\prime}\right)^{i-2 n} v(d)^{m-i+2 n} v\left(d^{\prime}\right)^{i-m-2 n}\right)^{\delta / 2} r_{i}\left(d, d^{\prime}\right) \phi,
$$

where

$$
p=\left(\begin{array}{ll}
a & b \\
0 & d
\end{array}\right) \in P_{i}^{2 n}, \quad p^{\prime}=\left(\begin{array}{ll}
a^{\prime} & 0 \\
c^{\prime} & d^{\prime}
\end{array}\right) \in \bar{P}_{i}^{m}, \quad \phi \in \mathscr{S}\left(G_{i}^{\prime}\right) .
$$

Let $\mathbb{1}_{j}$ denote the trivial representation of $G_{j}^{\prime}$. For $0 \leq i<m$ and an admissible representation $\pi$ of $G_{2 n}^{\prime}$, the Frobenius reciprocity gives

$$
\operatorname{Hom}_{G_{2 n}^{\prime} \times G_{m}^{\prime}}\left(S_{i} / S_{i+1}, \pi \otimes \mathbb{1}_{m}\right) \simeq \operatorname{Hom}_{M_{i}^{2 n} \times M_{i}^{m}}\left(\left(\pi^{\vee}\right)_{N_{i}^{2 n}} \otimes \delta_{P_{i}^{m}}^{1 / 2}, \mu_{i}^{\vee}\right),
$$

where $\delta_{P_{i}^{m}}$ is the modulus function on $P_{i}^{m}$ and $\left(\pi^{\vee}\right)_{N_{i}^{2 n}}$ is the normalized Jacquet module of $\pi^{\vee}$ associated to $P_{i}^{2 n}$. Since the quasicharacters of $G_{m-i}^{\prime}$ do not match, the space above is zero. Thus $\left(S_{i} / S_{i+1}\right)_{G_{m}^{\prime}}=0$, so that the natural map $\left(S_{m}\right)_{G_{m}^{\prime}} \rightarrow$ $\left(\omega_{2 n, m}\right)_{G_{m}^{\prime}}$ is surjective. If $\chi$ is a quasicharacter of $G_{m}^{\prime}$ and if a distribution $T$ on $\mathscr{S}\left(G_{m}^{\prime}\right)$ transforms according to $\chi$ under the action of $e \times G_{m}^{\prime}$, that is,

$$
T\left(r_{m}(e, h) f\right)=\chi(v(h)) T(f)
$$

for all $h \in G_{m}^{\prime}$, then there is a constant $c \in \mathbb{C}$ such that

$$
T(f)=c \int_{G_{m}^{\prime}} f(h) \chi(v(h)) d h, \quad f \in \mathscr{S}\left(G_{m}^{\prime}\right)
$$

(see Lemma 3.II.3 of [Mœglin et al. 1987]). It follows that

$$
\left(S_{m}\right)_{G_{m}^{\prime}} \simeq \operatorname{Ind}_{P_{m}^{2 n}}^{G_{2 n}^{\prime}}\left(\mathbb{1}_{2 n-m} \otimes \mathbb{1}_{m}\right) .
$$

Since Ind ${ }_{P_{m}^{2 n}}^{G_{2 n}^{\prime}}\left(\mathbb{1}_{2 n-m} \otimes \mathbb{1}_{m}\right)$ is irreducible as a representation of $G_{2 n}^{\prime}$ induced from a unitary representation [Sécherre 2009], we have

$$
\left(\omega_{\psi, V, 1}\right)_{H} \simeq \operatorname{Ind}_{P_{m}^{2 n}}^{G_{2 n}^{\prime}}\left(\mathbb{1}_{2 n-m} \otimes \mathbb{1}_{m}\right) .
$$

Thus the map from $\left(\omega_{\psi, V, 1}\right)_{H}$ to $R(V)$ is injective. This proves (1), (2).

In the $p$-adic case, Theorem 5.1 of [Mínguez 2009] tells us that $I\left(s_{0}\right)$ has a unique irreducible subrepresentation, which is $R(V)$, and hence $I\left(-s_{0}\right)$ has a unique irreducible quotient. We refer to [Lee 2007] for the archimedean analogue. From Lemma 1.1 we can infer that $f_{0}^{\left(-s_{0}\right)}$ generates $I\left(-s_{0}\right)$. It follows that $I\left(-s_{0}\right)=$ $R(U)$. The proof of (4) is complete. 
To prove (5), (6), it suffices to check that $b(s) M^{*}(s) f_{0}^{(s)}\left(\right.$ resp. $\left.M^{*}(s) f_{0}^{(s)}\right)$ are holomorphic and nonzero at $s=s_{0}$ (resp. $s=-s_{0}$ ) in light of [Kudla and Rallis 1988a, Proposition 4.9]. Let $\phi_{0}=\phi_{n, n} \in S\left(\mathrm{M}_{n}(\boldsymbol{D})\right)$ be as in the proof of Lemma 1.1. Define $\phi_{1} \in S\left(\mathrm{M}_{n, 2 n}(\boldsymbol{D})\right)$ by $\phi_{1}(x, y)=\phi_{0}(x) \phi_{0}(y)$. The sections $\mathfrak{F}_{\phi_{1}}^{(s)}$ and $\mathfrak{F}_{\hat{\phi}_{1}}^{(s)}$ are defined in the Appendix. Since $\mathfrak{F}_{\phi_{1}}^{(s)}$ is right $K$-invariant, so is $\mathfrak{F}_{\hat{\phi}_{1}}^{(s)}$ by Lemma A.1. From Propositions 10.7 and 10.8 of [Weil 1974], we know

$$
\mathfrak{F}_{\phi_{1}}^{(s)}=\mathfrak{F}_{\phi_{1}}^{(s)}(e) \cdot f_{0}^{(s)}=Z^{G J}\left(2 s+\frac{n^{\prime}}{2}, \phi_{0}, 1\right) \cdot f_{0}^{(s)}=f_{0}^{(s)} \prod_{j=1}^{n} \xi(2 s+\delta j)
$$

up to multiplication by exponential factors, where $\xi(s)=\zeta(s)$ in the $p$-adic case, and $\xi(s)=\Gamma(s)$ in the archimedean case. Observe that

$$
\begin{aligned}
\mathfrak{F}_{\hat{\phi}_{1}}^{(-s)} & =Z^{G J}\left(-2 s+\frac{n^{\prime}}{2}, \hat{\phi}_{0}, 1\right) \cdot f_{0}^{(s)} \\
& =(-1)^{n(\delta-1)} \gamma^{G J}\left(2 s-\frac{n^{\prime}-1}{2}, \mathbb{1}_{n}, \psi\right) Z^{G J}\left(2 s-\frac{n^{\prime}}{2}, \phi_{0}, 1\right) \cdot f_{0}^{(s)} .
\end{aligned}
$$

Substituting these into the equality in Lemma A.1, we get

$$
M(s) f_{0}^{(s)}=f_{0}^{(-s)} \prod_{j=1}^{n} \frac{\xi(2 s-\delta j+\delta)}{\xi(2 s+\delta j)} .
$$

Now we can easily conclude our proof.

\section{Proof of Theorem 1}

Back to the global setup, we write $\mathscr{A}$ for the space of automorphic forms on $G(\mathbb{A})$. For $\beta \in \operatorname{Her}_{n}(F)$ and $A \in \mathscr{A}$, let

$$
A_{\beta}(g)=\int_{\operatorname{Her}_{n}(F) \backslash \operatorname{Her}_{n}(\mathbb{A})} A(n(b) g) \psi(-\tau(\beta b)) d b, \quad g \in G(\mathbb{A})
$$

denote the $\beta$-th Fourier coefficient of $A$. The following lemma can be proven in exactly the same way as in [Kudla and Rallis 1990b; Tan 1999].

Lemma 2.1. Let $f^{(s)}$ be a holomorphic section of $I(s, \chi)$ and $\beta \in \operatorname{Her}_{n}(F)$ with $v(\beta) \neq 0$.

(1) $b(s, \chi) E_{\beta}\left(g ; f^{(s)}\right)$ is holomorphic in $\Re s>-\frac{1}{2}$.

(2) If $m \geq n$ and $\beta$ is represented by $V(F)$, then $E_{\beta}\left(g ; f_{\Phi}^{(s)}\right)$ can be made nonzero at $s=s_{0}$ for a suitable choice of $\Phi \in S\left(V^{n}(\mathbb{A})\right)$.

(3) If $\chi \rho(\chi)$ is not principal, then $E\left(g ; f^{(s)}\right)$ is entire. 
(4) If $\chi=\rho(\chi)^{-1}$, then the poles of $E\left(g ; f^{(s)}\right)$ in $\Re s>-\frac{1}{2}$ are at most simple and can only occur in the set

$$
\left\{\frac{n^{\prime}-j}{2} \mid j \in \mathbb{Z}, 0 \leq j<n^{\prime}, \chi^{0}=\epsilon_{E / F}^{j}\right\} .
$$

(5) If $\chi^{0}=\epsilon_{E / F}^{n^{\prime}+1}$, then $\left.E\left(g ; f^{(s)}\right)\right|_{s=0}$ is identically zero.

Definition 2.2. For each integer $l \leq n$, we say that $A \in \mathscr{A}$ has rank $\delta l$ if $A_{\beta}=0$ when $\operatorname{rank}_{D} \beta>l$, but $A_{\beta} \neq 0$ for some $\beta$ of rank $l$. When $\pi$ is a representation of $G(\mathbb{A})$ realized on a subspace of $\mathscr{A}$, we say that $\pi$ has rank at most $\delta l$ if all functions in $\pi$ have rank at most $\delta l$.

We call $A$ singular if it has rank less than $\delta n$. The following lemma can be proven in the same way as in the proof of [Howe 1981, Lemma 2.4].

Lemma 2.3. Let $\pi$ be a subrepresentation of $\mathscr{A}$. For every integer $l \leq n$ the following conditions are equivalent:

- $\pi$ has rank at most $\delta l$;

- for every place $v, G\left(F_{v}\right)$ acts on $\pi$ by a representation of rank at most $\delta l$;

- for at least one place $v, G\left(F_{v}\right)$ acts on $\pi$ by a representation of rank at most $\delta l$. In particular, if $G\left(F_{v}\right)$ acts on $\pi$ by a representation of rank at most $j$, then $G\left(F_{v}\right)$ acts on $\pi$ by a representation of rank at most $\delta \ell$, where $\ell=[j / \delta]$.

For $s^{\prime} \in \mathbb{C}$ with $\Re s^{\prime}>-\frac{1}{2}$, the residue $\operatorname{Res}_{s=s^{\prime}} E\left(g ; f^{(s)}\right)$ depends only on $f^{\left(s^{\prime}\right)}$, and $f^{\left(s^{\prime}\right)} \mapsto \operatorname{Res}_{s=s^{\prime}} E\left(g ; f^{(s)}\right)$ gives a $G(\mathbb{A})$ intertwining map

$$
A_{-1}\left(s^{\prime}\right): I\left(s^{\prime}, \chi\right) \rightarrow \mathscr{A} .
$$

Assume that $\chi=\rho(\chi)^{-1}$, assume that $j$ is an integer between 0 and $n^{\prime}$, assume that $\chi^{0}=\epsilon_{E / F}^{j}$, and assume that $j$ is not divisible by $\delta$. Let $s^{\prime}=\left(n^{\prime}-j\right) / 2$. To complete the proof of Theorem 1 , it remains to prove that $A_{-1}\left(s^{\prime}\right)$ is zero. Fix a finite inert place $v$ of $F$. By Theorem 1.2 of [Kudla and Sweet 1997], $I_{v}\left(s^{\prime}, \chi_{v}\right)$ has a unique irreducible submodule $R$ and

$$
I_{v}\left(s^{\prime}, \chi_{v}\right) / R \simeq \bigoplus_{V_{0}} R\left(V_{0}, \chi_{v}\right),
$$

where $V_{0}$ runs over all equivalence classes of hermitian spaces over $E_{v}$ of dimension $j$. Since the image of $A_{-1}\left(s^{\prime}\right)$ lies in the space of singular automorphic forms in view of Lemma 2.1(1) and since $R$ is nonsingular, the map $A_{-1}\left(s^{\prime}\right)$ factors through the quotient $\bigoplus_{V_{0}} R\left(V_{0}, \chi_{v}\right)$ at $v$. Proposition 1.4(3) shows that $G\left(F_{v}\right)$ acts on the image of $A_{-1}\left(s^{\prime}\right)$ by a representation of rank at most $j$. Put $\ell=[j / \delta]$. Lemma 2.3 shows that $G\left(F_{v}\right)$ acts on the image of $A_{-1}\left(s^{\prime}\right)$ by a representation of rank at most $\delta \ell$. Since $\delta \ell<j$, Proposition 1.4(3) forces $A_{-1}\left(s^{\prime}\right)$ to be zero. 


\section{Proof of Theorem 2}

Lemma 3.1. If $m=n$ or if $m-r>n$, then for all $\Phi \in S\left(V^{n}(\mathbb{A})\right)$ and $\beta \in \operatorname{Her}_{n}(F)$ with $v(\beta) \neq 0$,

$$
\left.E_{\beta}\left(g ; f_{\Phi}^{(s)}\right)\right|_{s=s_{0}}=\varkappa I_{\beta}(g ; \Phi) .
$$

Proof. The proof can be carried out by the same technique as in that of [Ichino 2004, Proposition 6.2]. We omit the details.

First we prove Theorem 2 in the case $m-r>n$. Ichino [2007] proved the special case of this result for $\delta=1$ (compare [Kudla and Rallis 1988b; Yamana 2013]). Many of the results there apply word for word in our general case.

If $m>2 n$, then $E\left(g ; f_{\Phi}^{\left(s_{0}\right)}\right)$ converges absolutely and the stated identity was proven by Weil [1965]. We may suppose that $m \leq 2 n$. Fix $\Phi^{0}=\bigotimes_{v} \Phi_{v}^{0} \in S\left(V^{n}(\mathbb{A})\right)$. By Theorem 10.6.2 of [Scharlau 1985], there is an inert place $w$ of $F$ such that the Witt index $r_{w}$ of $V_{w}$ satisfies $r_{w}<\delta(r+1)$, where $V_{w}$ stands for the hermitian space over $E_{w}$ corresponding to $V\left(F_{w}\right)$. Note that

$$
\delta m-r_{w}>\delta n .
$$

We consider the $G\left(F_{w}\right)$-intertwining map

$$
A_{-1, w}: S\left(V_{w}^{n^{\prime}}\right) \rightarrow \mathscr{A}, \quad \Phi_{w} \mapsto A_{-1}\left(s_{0}\right)\left(f_{\Phi}^{\left(s_{0}\right)}\right),
$$

where $\Phi=\Phi_{w} \otimes\left(\bigotimes_{v \neq w} \Phi_{v}^{0}\right)$. The invariant distribution theorem [Mœglin et al. 1987; Lee and Zhu 1998] asserts that $A_{-1, w}$ factors through the quotient $R\left(V_{w}, \chi_{V_{w}}\right)$. Lemma 2.1(1) shows that $A_{-1, w}\left(\Phi_{w}\right)$ is singular for every $\Phi_{w} \in S\left(V_{w}^{n^{\prime}}\right)$. If $w$ is finite, then $\delta m=2 r_{w}+2$ and $\delta n=r_{w}+1$, and hence $R\left(V_{w}, \chi_{V_{w}}\right)$ is irreducible and nonsingular by [Kudla and Sweet 1997, Theorem 1.2], so that $A_{-1, w}$ must be zero. If $w$ is real and $\nabla$ is the element of the universal enveloping algebra of the complexified Lie algebra of $G\left(F_{w}\right)$ defined by (2.1) of [Ichino 2007], then $\nabla A_{-1, w}\left(\Phi_{w}\right)=0$. Since Proposition 2.2 of [Ichino 2007] asserts that $\nabla f_{\Phi_{w}}^{\left(s_{0}\right)}$ generates the submodule $R\left(V_{w}, \chi_{V_{w}}\right)$ for a suitable choice of $\Phi_{w}$, the map $A_{-1, w}$ must be zero. Consequently, $E\left(g ; f_{\Phi}^{(s)}\right)$ is holomorphic at $s=s_{0}$ for every $\Phi \in S\left(V^{n}(\mathbb{A})\right)$.

Next we consider the $K_{w}$-intertwining map

$$
A_{w}: S\left(V_{w}^{n^{\prime}}\right) \rightarrow \mathscr{A},\left.\quad \Phi_{w} \mapsto E\left(g ; f_{\Phi}^{(s)}\right)\right|_{s=s_{0}}-I(g ; \Phi),
$$

where $\Phi=\Phi_{w} \otimes\left(\otimes_{v \neq w} \Phi_{v}^{0}\right)$. The image of $A_{w}$ lies in the space of singular automorphic forms by Lemma 3.1. We write $\mathscr{R}_{w}$ for the subspace of $\mathscr{A}$ spanned by residues $\operatorname{Res}_{s=s_{0}} E\left(g ; f^{(s)}\right)$, where $f^{(s)}$ is a holomorphic section of $I\left(s, \chi_{V}\right)$ of the form

$$
f^{(s)}=f_{w}^{(s)} \otimes\left(\bigotimes_{v \neq w} f_{\Phi_{v}^{0}}^{(s)}\right), \quad f_{w}^{(s)} \in I_{w}\left(s, \chi_{V_{w}}\right) .
$$


Then $A_{w}$ induces a $G\left(F_{w}\right)$-intertwining map $R\left(V_{w}, \chi_{V_{w}}\right) \rightarrow \mathscr{A} / \mathscr{R}_{w}$. The remaining part of the proof continues as in Section 3 of [Ichino 2007].

Theorem 2 is demonstrated in [Yamana 2011], provided that $\delta=1$ and $m \leq n$. Since the proof in our general case can be done by the same technique, we shall omit most of the details. We define the functions $a(s, \chi)$ and $b(s, \chi)$ by taking the complete Hecke $L$-functions in place of the local abelian $L$-factors in the definition of $a_{v}\left(s, \chi_{v}\right)$ and $b_{v}\left(s, \chi_{v}\right)$. We define a normalized global intertwining operator by

$$
M^{\circ}(s, \chi)=\frac{b(s, \chi)}{a(s, \chi)} M(s, \chi),
$$

which is holomorphic in $\Re s>-\frac{1}{2}$ by Lemma 1.2 and (1-2).

Let $\mathscr{C}=\left\{W_{v}\right\}$ be a collection of local hermitian spaces of dimension $m$ over $D_{v}$ such that $W_{v}$ is isometric to $V\left(F_{v}\right)$ for almost all $v$. We form a restricted tensor product $\Pi\left(\mathscr{b}, \chi_{V}\right)=\bigotimes_{v}^{\prime} R_{n^{\prime}}\left(W_{v}, \chi_{V_{v}}\right)$, which we can regard as a subrepresentation of $I\left(s_{0}, \chi_{V}\right)$. The proof of the following result is completely analogous to that of [Kudla and Rallis 1994, Theorem 3.1].

Proposition 3.2. Assume that $m \leq n$. Then

$$
\operatorname{dim} \operatorname{Hom}_{G(\mathbb{A})}\left(\Pi\left(\mathscr{C}, \chi_{V}\right), \mathscr{A}\right) \leq 1 .
$$

If there is no global hermitian space with $W_{v}$ as its completions, then

$$
\operatorname{dim} \operatorname{Hom}_{G(\mathbb{A})}\left(\Pi\left(\mathscr{C}, \chi_{V}\right), \mathscr{A}\right)=0 .
$$

Next we are going to prove the special case of Theorem 2 in which $m=n$. Let $\mathscr{C}=\left\{V\left(F_{v}\right)\right\}$. Since Proposition 1.4(2) shows that the two intertwining maps $\left.\Phi \mapsto E\left(g ; f_{\Phi}^{(s)}\right)\right|_{s=0}$ and $\Phi \mapsto I(g ; \Phi)$ define elements of the space

$$
\operatorname{Hom}_{G(\mathbb{A})}\left(\Pi\left(\mathscr{C}, \chi_{V}\right), \mathscr{A}\right),
$$

they must be proportional by Proposition 3.2. From Lemmas 2.1(2) and 3.1, they are nonvanishing, and the constant of proportionality is determined to be 2 .

We now suppose that $m<n$. Let $\mathscr{C}^{\prime}$ be a collection of local hermitian spaces of dimension $2 n-m$ obtained by adding a split space of suitable dimension to $\mathscr{C}$. By Proposition 1.4(4) and (5), $\Pi\left(\mathscr{C}^{\prime}, \chi_{V}\right)$ has a unique irreducible quotient $\Pi\left(\mathscr{C}, \chi_{V}\right)$, and $M^{\circ}\left(-s_{0}, \chi_{V}\right)$ induces a nonzero intertwining map $\Pi\left(\mathscr{C}^{\prime}, \chi_{V}\right) \rightarrow \Pi\left(\mathscr{C}, \chi_{V}\right)$. The same reasoning as in Section 4 of [Yamana 2011] implies the following result:

Proposition 3.3. Suppose that $m<n$. Let $f^{(s)}$ be a standard section of $I\left(s, \chi_{V}\right)$ such that $f^{\left(s_{0}\right)} \in \Pi\left(\mathscr{C}, \chi_{V}\right)$. Put $h^{(-s)}=M^{\circ}\left(s, \chi_{V}\right) f^{(s)}$.

(1) $E\left(g ; f^{(s)}\right)$ is holomorphic at $s=s_{0}$. 
(2) $h^{(s)}$ is holomorphic at $s=-s_{0}, h^{\left(-s_{0}\right)} \in \Pi\left(\mathscr{C}^{\prime}, \chi_{V}\right)$, and

$$
\operatorname{Res}_{s=-s_{0}} E\left(g ; h^{(s)}\right)=-\left.\operatorname{Res}_{s=s_{0}}\left[\frac{b\left(s, \chi_{V}\right)}{a\left(s, \chi_{V}\right)}\right] E\left(g ; f^{(s)}\right)\right|_{s=s_{0}} .
$$

Lemma 3.4. If $m<n$, then the image of the map $A_{-1}\left(-s_{0}\right)$ lies in the space of square integrable automorphic forms on $G(\mathbb{A})$.

Proof. We use [Kudla and Sweet 1997, Proposition 6.2] and follow closely the guideline of the proof of [Kudla and Rallis 1994, Proposition 4.6].

Proposition 3.5. If $m<n$, then the restriction of $A_{-1}\left(-s_{0}\right)$ to $\Pi\left(\mathscr{C}^{\prime}, \chi_{V}\right)$ is zero unless $\mathscr{C}$ is the set of localizations of a global space, in which case it defines a nonzero intertwining map $\Pi\left(\mathscr{b}, \chi_{V}\right) \rightarrow \mathscr{A}$.

Proof. The image of $A_{-1}\left(-s_{0}\right)$ is completely reducible in view of Lemma 3.4. Thus the restriction of $A_{-1}\left(-s_{0}\right)$ to $\Pi\left(\mathscr{C}^{\prime}, \chi_{V}\right)$ must factor through the unique irreducible quotient $\Pi\left(\mathscr{C}, \chi_{V}\right)$. Proposition 3.2 shows that $\Pi\left(\mathscr{C}, \chi_{V}\right)$ makes no contribution unless $\mathscr{C}$ comes from a global space. It remains to check that $A_{-1}\left(-s_{0}\right)$ is nonzero on $\Pi\left(V, \chi_{V}\right)$. From Proposition 3.3(2) this amounts to proving that the holomorphic value $\left.E\left(g ; f_{\Phi}^{(s)}\right)\right|_{s=s_{0}}$ is nonzero for a good choice of $\Phi \in S\left(V^{n}(\mathbb{A})\right)$.

Let $\beta_{0} \in \operatorname{Her}_{m}(F)$ with $v\left(\beta_{0}\right) \neq 0$. Put

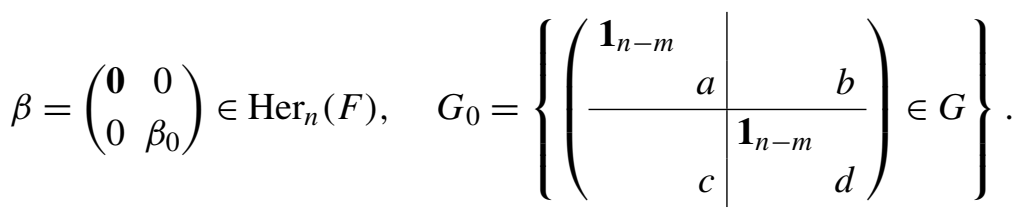

Define $\Phi_{0} \in S\left(V^{m}(\mathbb{A})\right)$ by $\Phi_{0}(y)=\Phi((0, y))$ for $y \in V^{m}(\mathbb{A})$. The nonvanishing can be proven by considering the $\beta$-th Fourier coefficient of $E\left(g ; f_{\Phi}^{(s)}\right)$ as in Section 6 of [Yamana 2011] (compare Theorem 4.9 of [Kudla and Rallis 1994]). The exponents of the $n-m+1$ terms in this Fourier coefficient are distinct at $s=s_{0}$, so that there can be no cancellations among them. The first term is just the $\beta_{0}$-th Fourier coefficient of the central value of the Eisenstein series on $G_{0}(\mathbb{A})$ attached to the standard section $f_{\Phi_{0}}^{(s)}$. Lemma 2.1(2) now completes our proof.

Corollary 3.6. Suppose that $m \leq n$. Let $f^{(s)}$ be a standard section of $I\left(s, \chi_{V}\right)$ such that $f^{\left(s_{0}\right)} \in \Pi\left(\mathscr{C}, \chi_{V}\right)$. If $\mathscr{C}$ cannot be the set of localizations of any global space, then $\left.E\left(g ; f^{(s)}\right)\right|_{s=s_{0}}$ is identically zero.

Proof. Propositions 3.2, 3.3(2) and 3.5 prove this corollary.

The regularized Siegel-Weil formula can be deduced from Propositions 3.2 and 3.5. 
Theorem 3.7. Assume that $m<n$. Then there is a nonzero constant $c_{0}$ such that if holomorphic sections $f^{(s)}$ of $I\left(s, \chi_{V}\right)$ and $\Phi \in S\left(V^{n}(\mathbb{A})\right)$ satisfy the relation

$$
M^{\circ}\left(-s_{0}, \chi_{V}\right) f^{\left(-s_{0}\right)}=f_{\Phi}^{\left(s_{0}\right)},
$$

then we have

$$
\operatorname{Res}_{s=-s_{0}} E\left(g ; f^{(s)}\right)=c_{0} I(g ; \Phi) .
$$

Finally, we prove Theorem 2 when $m<n$. Applying Proposition 3.3(2) and Theorem 3.7 to $h^{(-s)}=M^{\circ}\left(s, \chi_{V}\right) f_{\Phi}^{(s)}$, we see that

$$
\left.E\left(g ; f_{\Phi}^{(s)}\right)\right|_{s=s_{0}}=c I(g ; \Phi),
$$

where $c$ is independent of $\Phi$. One can prove that $c=2$ in exactly the same manner as in Section 6 of [Yamana 2011].

\section{Appendix. Zeta integrals for $\mathrm{GL}_{n}(D)$}

Let $F$ be a local field of characteristic zero and $\boldsymbol{D}$ a division algebra central and of dimension $\delta^{2}$ over $F$. We begin by reviewing the Godement-Jacquet construction of the local factors of representations of $G_{n}^{\prime}=\mathrm{GL}_{n}(\boldsymbol{D})$. The Fourier transform $\hat{\phi} \in \mathscr{S}\left(\mathbf{M}_{b a}(\boldsymbol{D})\right)$ of $\phi \in \mathscr{S}\left(\mathbf{M}_{a b}(\boldsymbol{D})\right)$ is defined by

$$
\hat{\phi}(x)=\int_{\mathbf{M}_{a b}(\boldsymbol{D})} \phi(y) \psi(\tau(x y)) d y, \quad x \in \mathbf{M}_{b a}(\boldsymbol{D}),
$$

where the Haar measure $d y$ is so chosen that

$$
\int_{\mathrm{M}_{a b}(\boldsymbol{D})} \hat{\phi}\left({ }^{t} y\right) d y=\phi(0) .
$$

In the archimedean case $S\left(\mathbf{M}_{a b}(\boldsymbol{D})\right)$ is the subspace of $\mathscr{S}\left(\mathbf{M}_{a b}(\boldsymbol{D})\right)$ as defined on p. 115 of [Godement and Jacquet 1972], and in the $p$-adic case $S\left(\mathbf{M}_{a b}(\boldsymbol{D})\right)=$ $\mathscr{S}\left(\mathrm{M}_{a b}(\boldsymbol{D})\right)$.

Let $\pi$ be an irreducible admissible representation of $G_{n}^{\prime}$. We write $\pi^{\vee}$ for its admissible dual and denote the standard pairing on $\pi^{\vee} \otimes \pi$ by $\langle$,$\rangle . For s \in \mathbb{C}$, $\phi \in \mathscr{S}\left(\mathrm{M}_{n}(\boldsymbol{D})\right), \xi \in \pi$ and $\xi^{\vee} \in \pi^{\vee}$ we set

$$
Z^{G J}\left(s, \phi, \xi \otimes \xi^{\vee}\right)=\int_{G_{n}^{\prime}}\left\langle\pi(g) \xi, \xi^{\vee}\right\rangle \phi(g)|v(g)|_{F}^{s+n^{\prime} / 2} d g .
$$

This integral converges in some half-plane and extends to a meromorphic function on the whole $s$-plane satisfying

$$
Z^{G J}\left(-s, \hat{\phi}, \xi^{\vee} \otimes \xi\right)=(-1)^{n(\delta-1)} \gamma^{G J}\left(s+\frac{1}{2}, \pi, \psi\right) Z^{G J}\left(s, \phi, \xi \nabla \xi^{\vee}\right) .
$$


Fix a pair $\chi=\left(\chi_{1}, \chi_{2}\right)$ of quasicharacters of $F^{\times}$. Recall $\chi^{0}=\chi_{1} \chi_{2}$. We attach a section $s \mapsto \mathfrak{F}_{\phi}^{(s, \chi)}$ to each $\phi \in \mathscr{S}\left(\mathrm{M}_{n, 2 n}(\boldsymbol{D})\right)$ by setting

$$
\mathfrak{F}_{\phi}^{(s, \chi)}(g)=\chi_{1}(v(g))|v(g)|_{F}^{s+n^{\prime} / 2} \int_{G_{n}^{\prime}} \phi((0, t) g) \chi^{0}(v(t))|v(t)|_{F}^{2 s+n^{\prime}} d t .
$$

This integral converges absolutely for sufficiently large $\Re s$. Observe that if $\phi$ belongs to $S\left(\mathrm{M}_{n, 2 n}(\boldsymbol{D})\right)$, then $\mathfrak{F}_{\phi}^{(s, \chi)} \in I(s, \chi)$ (compare (1-1)). For $\varphi \in \mathscr{S}\left(\mathrm{M}_{2 n, n}(\boldsymbol{D})\right)$ we define a section $\mathfrak{F}_{\varphi}^{(s, \chi)}$ of $I(s, \chi)$ to be

$$
\chi_{2}(v(g))^{-1}|v(g)|_{F}^{-s-n^{\prime} / 2} \int_{G_{n}^{\prime}} \varphi\left(g^{-1}\left(\begin{array}{l}
t \\
0
\end{array}\right)\right) \chi^{0}(v(t))|v(t)|_{F}^{2 s+n^{\prime}} d t .
$$

Lemma A.1. For each $\phi \in S\left(\mathrm{M}_{n, 2 n}(\boldsymbol{D})\right)$,

$$
M(s, \chi) \mathfrak{F}_{\phi}^{(s, \chi)}=\frac{(-1)^{n(\delta-1)} \chi_{1}(-1)^{n^{\prime}}}{\gamma^{G J}\left(2 s-\frac{n^{\prime}-1}{2}, \chi^{0} \circ v_{n}, \psi\right)} \mathfrak{F}_{\hat{\phi}}^{\left(-s, \rho(\chi)^{-1}\right)} .
$$

Proof. The case $n=\delta=1$ is discussed in Lemma 14.7.1 of [Jacquet 1972]. The proof is substantially the same. For $g \in G_{2 n}^{\prime}$ we put

$$
\Psi_{g}(t)=\int_{\mathrm{M}_{n}(\boldsymbol{D})} \phi((t, x) g) d x
$$

for $t \in \mathrm{M}_{n}(\boldsymbol{D})$. Then

$$
\begin{aligned}
& M(s, \chi) \mathfrak{F}_{\phi}^{(s, \chi)}(g) \\
& =\int_{\mathrm{M}_{n}(\boldsymbol{D})} \mathfrak{F}_{\phi}^{(s, \chi)}\left(\left(\begin{array}{cc}
0 & \mathbf{1}_{n} \\
\mathbf{1}_{n} & 0
\end{array}\right)\left(\begin{array}{cc}
\mathbf{1}_{n} & x \\
0 & \mathbf{1}_{n}
\end{array}\right) g\right) d x \\
& =\chi_{1}\left((-1)^{n^{\prime}} v(g)\right)|v(g)|_{F}^{s+n^{\prime} / 2} \\
& \quad \times \int_{\mathrm{M}_{n}(\boldsymbol{D})} \int_{G_{n}^{\prime}} \phi\left((0, t)\left(\begin{array}{cc}
0 & \mathbf{1}_{n} \\
\mathbf{1}_{n} & x
\end{array}\right) g\right) \chi^{0}(v(t))|v(t)|_{F}^{2 s+n^{\prime}} d t d x \\
& =\chi_{1}\left((-1)^{n^{\prime}} v(g)\right)|v(g)|_{F}^{s+n^{\prime} / 2} \int_{\mathrm{M}_{n}(\boldsymbol{D})} \int_{G_{n}^{\prime}} \phi((t, x) g) \chi^{0}(v(t))|v(t)|_{F}^{2 s} d t d x \\
& =\chi_{1}(-1)^{n^{\prime}} \chi_{1}(v(g))|v(g)|_{F}^{s+n^{\prime} / 2} Z^{G J}\left(2 s-\frac{n^{\prime}}{2}, \Psi_{g}, \chi^{0} \circ v_{n}\right) .
\end{aligned}
$$

Since $\widehat{\Psi_{g}}(t)=|v(g)|_{F}^{-n^{\prime}} \hat{\phi}\left(g^{-1}\left(\begin{array}{l}t \\ 0\end{array}\right)\right)$,

$$
\chi_{1}(v(g))|v(g)|_{F}^{s+n^{\prime} / 2} Z^{G J}\left(\frac{n^{\prime}}{2}-2 s, \widehat{\Psi_{g}},\left(\chi^{0} \circ v_{n}\right)^{-1}\right)=\mathfrak{F}_{\hat{\phi}}^{\left(-s, \rho(\chi)^{-1}\right)} .
$$

Lemma A.1 follows from the functional equation of $Z^{G J}\left(s, \phi, \chi^{0} \circ v_{n}\right)$. 
Fix $A \in \mathrm{GL}_{n}(\boldsymbol{D})$. For a section $f^{(s)}$ of $I(s, \chi)$, the integral

$$
l_{A}\left(f^{(s)}\right)=\int_{\mathrm{M}_{n}(\boldsymbol{D})} f^{(s)}\left(\left(\begin{array}{cc}
\mathbf{1}_{n} & 0 \\
x & \mathbf{1}_{n}
\end{array}\right)\right) \psi(\tau(A x)) d x
$$

converges absolutely for $\Re s \gg 0$. In the $p$-adic case, Karel [1979] has proven that $l_{A}\left(f^{(s)}\right)$ admits an entire analytic continuation to the whole $s$-plane and satisfies a functional equation

$$
l_{A} \circ M(s, \chi)=\chi^{0}(v(A))^{-1}|v(A)|_{F}^{-2 s} c(s, \chi, \psi) l_{A}
$$

for some meromorphic function $c(s, \chi, \psi)$. The factor $c(s, \chi, \psi)$ is independent of the choice of $A$. Analogous results are proven in the archimedean case in [Wallach 1988]. The normalization $M^{\dagger}(s, \chi)$ of $M(s, \chi)$ is defined so that

$$
l_{A} \circ M^{\dagger}(s, \chi)=\chi_{2}(-1)^{n^{\prime}} \chi^{0}(v(A))^{-1}|v(A)|_{F}^{-2 s} l_{A} .
$$

Lemma A.2. For each $\Phi \in S\left(\mathrm{M}_{n, 2 n}(\boldsymbol{D})\right)$,

$$
M^{\dagger}(s, \chi) \mathfrak{F}_{\Phi}^{(s, \chi)}=\chi_{2}(-1)^{n^{\prime}} \mathfrak{F}_{\hat{\Phi}}^{\left(-s, \rho(\chi)^{-1}\right)} .
$$

Proof. It is enough to show that

$$
l_{A}\left(\mathfrak{F}_{\hat{\Phi}}^{\left(-s, \rho(\chi)^{-1}\right)}\right)=\chi^{0}(v(A))^{-1}|\nu(A)|_{F}^{-2 s} l_{A}\left(\mathfrak{F}_{\Phi}^{(s, \chi)}\right) .
$$

Take $\phi_{1}, \phi_{2} \in S\left(\mathrm{GL}_{n}(\boldsymbol{D})\right)$ and define $\Phi \in S\left(\mathrm{M}_{n, 2 n}(\boldsymbol{D})\right)$ by $\Phi(x, y)=\hat{\phi}_{1}(x) \phi_{2}(y)$. Then

$$
\begin{aligned}
l_{A}\left(\mathfrak{F}_{\Phi}^{(s, \chi)}\right) & =\int_{\mathrm{M}_{n}(\boldsymbol{D})} \mathfrak{F}_{\Phi}^{(s, \chi)}\left(\left(\begin{array}{cc}
\mathbf{1}_{n} & 0 \\
x & \mathbf{1}_{n}
\end{array}\right)\right) \psi(\tau(A x)) d x \\
& =\int_{\mathrm{M}_{n}(\boldsymbol{D})} \int_{\mathrm{GL}_{n}(\boldsymbol{D})} \Phi\left((0, t)\left(\begin{array}{cc}
\mathbf{1}_{n} & 0 \\
x & \mathbf{1}_{n}
\end{array}\right)\right) \chi^{0}(v(t))|v(t)|_{F}^{2 s+n^{\prime}} d t \psi(\tau(A x)) d x \\
& =\int_{\mathrm{GL}_{n}(\boldsymbol{D})} \phi_{1}\left(-A t^{-1}\right) \phi_{2}(t) \chi^{0}(v(t))|v(t)|_{F}^{2 s} d t .
\end{aligned}
$$

Similarly, $l_{A}\left(\mathfrak{F}_{\hat{\Phi}}^{\left(-s, \rho(\chi)^{-1}\right)}\right)$ is equal to

$$
\begin{aligned}
\int_{\mathrm{M}_{n}(\boldsymbol{D})} \int_{\mathrm{GL}_{n}(\boldsymbol{D})} \phi_{1}(-t) \hat{\phi}_{2}(-x t) \chi^{0}(v(t))^{-1}|v(t)|_{F}^{-2 s+n^{\prime}} \psi(\tau(A x)) d t d x \\
=\int_{\mathrm{GL}_{n}(\boldsymbol{D})} \phi_{1}(-t) \phi_{2}\left(t^{-1} A\right) \chi^{0}(v(t))^{-1}|v(t)|_{F}^{-2 s} d t \\
=\chi^{0}(v(A))^{-1}|v(A)|_{F}^{-2 s} l_{A}\left(\mathfrak{F}_{\Phi}^{(s, \chi)}\right) .
\end{aligned}
$$

Since both $l_{A}\left(\mathfrak{F}_{\Phi}^{(s, \chi)}\right)$ and $l_{A}\left(\mathfrak{F}_{\hat{\Phi}}^{\left(-s, \rho(\chi)^{-1}\right)}\right)$ are not identically zero for a suitable choice of $\phi_{1}$ and $\phi_{2}$, the proof is complete. 
The embedding $i$ of $G_{n}^{\prime} \times G_{n}^{\prime}$ into $G_{2 n}^{\prime}$ is given by

$$
\left(g_{1}, g_{2}\right) \mapsto w_{1}\left(\begin{array}{cc}
g_{1} & 0 \\
0 & g_{2}
\end{array}\right) w_{1}^{-1}, \quad w_{1}=\left(\begin{array}{cc}
2^{-1} \cdot \mathbf{1}_{n} & -2^{-1} \cdot \mathbf{1}_{n} \\
\mathbf{1}_{n} & \mathbf{1}_{n}
\end{array}\right) .
$$

Let $\pi$ be an irreducible admissible representation of $G_{n}^{\prime}$. For $\xi \in \pi, \xi^{\vee} \in \pi^{\vee}$ and a section $f^{(s)}$ of $I(s, \chi)$, we define the zeta integral by

$$
Z\left(\xi \otimes \xi^{\vee}, f^{(s)}\right)=\int_{G_{n}^{\prime}}\left\langle\pi(g) \xi, \xi^{\vee}\right\rangle f^{(s)}(i(g, e)) d g,
$$

following [Piatetski-Shapiro and Rallis 1987a; Lapid and Rallis 2005]. This integral converges absolutely for $\Re s \gg 0$ and extends to a meromorphic function in $s$ that satisfies the functional equation

$$
Z\left(\xi \otimes \xi^{\vee}, M^{\dagger}(s, \chi) f^{(s)}\right)=\pi(-1) \gamma\left(s+\frac{1}{2}, \pi \times \chi, \psi\right) Z\left(\xi \otimes \xi^{\vee}, f^{(s)}\right) .
$$

Lapid and Rallis [2005] demonstrated the special case of the following result for $\delta=1$ in a different manner. It was pointed out by Wee Teck Gan [2012] that there is a typo in [Lapid and Rallis 2005, (25)].

Proposition A.3. For any irreducible admissible representation $\pi$ of $G_{n}^{\prime}$ and any pair $\chi=\left(\chi_{1}, \chi_{2}\right)$ of quasicharacters of $F^{\times}$,

$$
\gamma(s, \pi \times \chi, \psi)=\gamma^{G J}\left(s, \pi \otimes \chi_{1}, \psi\right) \gamma^{G J}\left(s, \pi^{\vee} \otimes \chi_{2}, \psi\right) .
$$

Proof. Let $\mathscr{F}_{\Phi}^{(s, \chi)}$ be the translate of $\mathfrak{F}_{\Phi}^{(s, \chi)}$ by the element $w_{1} \in G_{2 n}^{\prime}$. Then $Z\left(\xi \otimes \xi^{\vee}, \mathscr{F}_{\Phi}^{(s, \chi)}\right)$

$$
\begin{aligned}
= & \int_{G_{n}^{\prime}}\left\langle\pi(g) \xi, \xi^{\vee}\right\rangle \chi_{1}(v(g))|v(g)|_{F}^{s+n^{\prime} / 2} \\
& \times \int_{G_{n}^{\prime}} \Phi\left((0, t) w_{1}\left(\begin{array}{cc}
g & 0 \\
0 & \mathbf{1}_{n}
\end{array}\right)\right) \chi^{0}(v(t))|v(t)|_{F}^{2 s+n^{\prime}} d t d g \\
= & \int_{G_{n}^{\prime} \times G_{n}^{\prime}}\left\langle\left(\pi \otimes \chi_{1}\right)(g) \xi,\left(\pi^{\vee} \otimes \chi_{2}\right)(t) \xi^{\vee}|v(g t)|_{F}^{s+n^{\prime} / 2} \Phi(g, t) d g d t .\right.
\end{aligned}
$$

If $\Phi(x, y)$ is of the form $\phi_{1}(x) \phi_{2}(y)$, then the last integral is equal to

$$
\left\langle Z^{G J}\left(s, \pi \otimes \chi_{1}, \phi_{1}\right) \xi, Z^{G J}\left(s, \pi^{\vee} \otimes \chi_{2}, \phi_{2}\right) \xi^{\vee}\right\rangle .
$$

Piatetski-Shapiro and Rallis [1987a] employ this relation to calculate the unramified local zeta integrals. 
We can see by Lemma A.2 that

$$
\begin{aligned}
Z\left(\xi \otimes \xi^{\vee}, M^{\dagger}(s, \chi) \mathscr{F}_{\Phi}^{(s, \chi)}\right)=\chi_{2}(-1)^{n^{\prime}} \int_{G_{n}^{\prime} \times G_{n}^{\prime}} \hat{\phi}_{1}(g) \hat{\phi}_{2}(t) \\
\quad \times|v(g t)|_{F}^{-s+n^{\prime} / 2}\left\{\left(\pi \otimes \chi_{1}\right)\left(g^{-1}\right) \xi,\left(\pi^{\vee} \otimes \chi_{2}\right)\left(-t^{-1}\right) \xi^{\vee}\right\rangle d g d t .
\end{aligned}
$$

The stated relation follows upon combining these with the definitions of the gamma factors.

$$
\begin{aligned}
& \text { Let } \chi=1 \text {. Put } \Delta_{s}(g)=f_{0}^{\left(s-n^{\prime} / 2\right)}\left(w_{1}\left(\begin{array}{ll}
g & \\
& \mathbf{1}_{n}
\end{array}\right)\right) \text { for } g \in G_{n}^{\prime} \text {. Note that } \\
& \Delta_{s}\left(k_{1} g k_{2}\right)=f_{0}^{\left(s-n^{\prime} / 2\right)}\left(w_{1}\left(\begin{array}{ll}
k_{1} g k_{2} & \\
& \mathbf{1}_{n}
\end{array}\right)\right) \\
& =f_{0}^{\left(s-n^{\prime} / 2\right)}\left(i\left(k_{1}, k_{1}\right) w_{1}\left(\begin{array}{ll}
g & \\
& \mathbf{1}_{n}
\end{array}\right)\left(\begin{array}{cc}
k_{2} & \\
& k_{1}^{-1}
\end{array}\right)\right)=\Delta_{s}(g)
\end{aligned}
$$

for $k_{1}, k_{2} \in K_{n}$ and $g \in G_{n}^{\prime}$. An explicit formula for this function is obtained in [Piatetski-Shapiro and Rallis 1987a, Proposition 6.4] in the case of symplectic or split even orthogonal groups. One can deduce from their argument a formula of the same type for the unit groups of simple algebras.

Lemma A.4. (1) If $F$ is a p-adic field and $g=k_{1} d k_{2}$ with elements $k_{1}, k_{2} \in K_{n}$ and $d=\operatorname{diag}\left[\varpi^{a_{1}}, \ldots, \varpi^{a_{n}}\right]$, where $\varpi$ is a generator of the maximal ideal of $\mathcal{O}$, and we put $q=|v(\varpi)|_{F}^{-1}$, then

$$
\Delta_{s}(g)=q^{-s \sum_{i=1}^{n}\left|a_{i}\right|}
$$

(2) Assume that $F=\mathbb{R}$ or $\mathbb{C}$. Put $t=[F: \mathbb{R}]$. If $g=k_{1} d k_{2}$ with $k_{1}, k_{2} \in K_{n}$ and $d=\operatorname{diag}\left[d_{1}, \ldots, d_{n}\right]$ with positive real numbers $d_{i}$, then

$$
\Delta_{s}(g)=2^{n \delta t s} \prod_{i=1}^{n}\left(d_{i}^{-1}+d_{i}\right)^{-\delta t s} .
$$

Lemma A.5. If $\Re s>\delta(n-1)$, then $\Delta_{s}$ belongs to $L^{1}\left(G_{n}^{\prime}\right)$. 
Proof. Put $\sigma=\Re$ s. We consider the $p$-adic case. Proposition 1.5.2 of [Casselman 1995] gives a positive constant $c$ such that

$$
\begin{aligned}
\int_{G_{n}^{\prime}}\left|\Delta_{s}(g)\right| d g & \leq c \sum_{a_{1} \geq a_{2} \geq \cdots \geq a_{n}} q^{-\sigma \sum_{i=1}^{n}\left|a_{i}\right|} \prod_{j=1}^{n} q^{\delta(n+1-2 j) a_{j}} \\
& \leq c \prod_{j=1}^{n} \sum_{a_{j} \in \mathbb{Z}} q^{-\sigma\left|a_{j}\right|+\delta(n+1-2 j) a_{j}} \\
& =c \prod_{j=1}^{n}\left(\frac{1}{1-q^{\delta(n+1-2 j)-\sigma}}+\frac{q^{\delta(2 j-n-1)-\sigma}}{1-q^{\delta(2 j-n-1)-\sigma}}\right) .
\end{aligned}
$$

The archimedean case can be proven in the same way.

Lemma A.6. If $\sigma>0$, then the function $z \mapsto \Delta_{\sigma}(z g)$ is integrable over the center $Z$ of $G_{n}^{\prime}$ for any $g \in G_{n}^{\prime}$. Moreover, there exists a positive constant $A_{\sigma}$ depending only on $\sigma$ such that, for every $g \in G_{n}^{\prime}$,

Proof. In the $p$-adic case,

$$
\int_{Z} \Delta_{\sigma}(z g) d z \leq A_{\sigma}
$$

$$
\int_{Z} \Delta_{\sigma}(z g) d z=\sum_{j \in \mathbb{Z}} q^{-\sigma \sum_{i=1}^{n}\left|a_{i}+\delta j\right|} \leq \sum_{j \in \mathbb{Z}} q^{-\sigma|j|}=\frac{1+q^{-\sigma}}{1-q^{-\sigma}} .
$$

The proof for the archimedean case is completely analogous.

Recall that $\pi$ is called square integrable if it admits a unitary central character and its matrix coefficients are square integrable modulo the center. For $\left(s_{1}, s_{2}\right) \in \mathbb{C}$, we write $I\left(s_{1}, s_{2}\right)=I\left(0,\left(\alpha_{F}^{s_{1}}, \alpha_{F}^{s_{2}}\right)\right)$.

Proposition A.7. If $\pi$ is square integrable, $\Re s_{1}, \Re s_{2}>-\delta / 2$ and $f \in I\left(s_{1}, s_{2}\right)$, then the integral defining $Z\left(\xi \otimes \xi^{\vee}, f\right)$ is absolutely convergent.

Proof. Put $\sigma=\min \left\{\Re s_{1}, \Re s_{2}\right\}$. Note that $\left(\alpha_{F} \circ v_{2 n}\right)^{s^{\prime}} \cdot f_{0}^{(s)} \in I\left(s+s^{\prime}, s-s^{\prime}\right)$. By Lemma A.4, we can majorize $|f((g, e))|$ by $c f_{0}^{(\sigma)}((g, e))$ for some positive constant $c$. Our task is to check that for any $\sigma>-\delta / 2$,

$$
\int_{G_{n}^{\prime}}\left|\left\langle\pi(g) \xi, \xi^{\vee}\right\rangle\right| \Delta_{\sigma+n^{\prime} / 2}(g) d g
$$

is finite. Take a constant $\sigma^{\prime}$ so that $0<\sigma^{\prime}<\sigma+\delta / 2$. The square of this integral is less than or equal to the product of the integrals

$$
\int_{G_{n}^{\prime}} \Delta_{2 \sigma+n^{\prime}-2 \sigma^{\prime}}(z g) d g
$$


and

$$
\begin{aligned}
\int_{G_{n}^{\prime}}\left|\left\langle\pi(g) \xi, \xi^{\vee}\right\rangle\right|^{2} \Delta_{2 \sigma^{\prime}}(g) d g & =\int_{Z \backslash G_{n}^{\prime}}\left|\left\langle\pi(\dot{g}) \xi, \xi^{\vee}\right\rangle\right|^{2} \int_{Z} \Delta_{2 \sigma^{\prime}}(z \dot{g}) d z d \dot{g} \\
& =A_{2 \sigma^{\prime}} \int_{Z \backslash G_{n}^{\prime}}\left|\left\langle\pi(\dot{g}) \xi, \xi^{\vee}\right\rangle\right|^{2} d \dot{g},
\end{aligned}
$$

both of which are finite, the first by Lemma A.5 and the second by Lemma A.6.

\section{Acknowledgements}

We would like to thank the National University of Singapore, where this paper was partly written.

\section{References}

[Casselman 1995] W. Casselman, "Introduction to the theory of admissible representations of p-adic reductive groups", preprint, 1995.

[Gan 2000] W. T. Gan, "A Siegel-Weil formula for exceptional groups", J. Reine Angew. Math. 528 (2000), 149-181. MR 2001k:11089 Zbl 1005.11022

[Gan 2012] W. T. Gan, "Doubling zeta integrals and local factors for metaplectic groups", Nagoya Math. J. 208 (2012), 67-95. MR 3006697 Zbl 06127268

[Gan and Takeda 2011] W. T. Gan and S. Takeda, "On the regularized Siegel-Weil formula (the second term identity) and non-vanishing of theta lifts from orthogonal groups", J. Reine Angew. Math. 659 (2011), 175-244. MR 2012i:22029 Zbl 05971444

[Godement and Jacquet 1972] R. Godement and H. Jacquet, Zeta functions of simple algebras, Lecture Notes in Mathematics 260, Springer, Berlin, 1972. MR 49 \#7241 Zbl 0244.12011

[Howe 1981] R. Howe, "Automorphic forms of low rank", pp. 211-248 in Noncommutative harmonic analysis and Lie groups (Marseille, 1980), edited by J. Carmona and M. Vergne, Lecture Notes in Math. 880, Springer, Berlin, 1981. MR 83j:10033 Zbl 0463.10015

[Ichino 2004] A. Ichino, "A regularized Siegel-Weil formula for unitary groups", Math. Z. 247:2 (2004), 241-277. MR 2005g:11082 Zbl 1071.11022

[Ichino 2007] A. Ichino, "On the Siegel-Weil formula for unitary groups", Math. Z. 255:4 (2007), 721-729. MR 2007m:11063 Zbl 1223.11056

[Jacquet 1972] H. Jacquet, Automorphic forms on GL(2), II, Lecture Notes in Mathematics 278, Springer, Berlin, 1972. MR 58 \#27778 Zbl 0243.12005

[Karel 1979] M. L. Karel, "Functional equations of Whittaker functions on p-adic groups", Amer. J. Math 101:6 (1979), 1303-1325. MR 81b:10021 Zbl 0427.22011

[Kudla 1994] S. S. Kudla, "Splitting metaplectic covers of dual reductive pairs", Israel J. Math. 87:1-3 (1994), 361-401. MR 95h:22019 Zbl 0840.22029

[Kudla and Rallis 1988a] S. S. Kudla and S. Rallis, "On the Weil-Siegel formula", J. Reine Angew. Math. 387 (1988), 1-68. MR 90e:11059 Zbl 0644.10021

[Kudla and Rallis 1988b] S. S. Kudla and S. Rallis, "On the Weil-Siegel formula, II: The isotropic convergent case”, J. Reine Angew. Math. 391 (1988), 65-84. MR 90e:11060 Zbl 0644.10022

[Kudla and Rallis 1990a] S. S. Kudla and S. Rallis, "Degenerate principal series and invariant distributions", Israel J. Math. 69:1 (1990), 25-45. MR 91e:22016 Zbl 0708.22005 
[Kudla and Rallis 1990b] S. S. Kudla and S. Rallis, "Poles of Eisenstein series and $L$-functions", pp. 81-110 in Festschrift in honor of I. I. Piatetski-Shapiro on the occasion of his sixtieth birthday, 2 (Tel Aviv, 1989), edited by S. Gelbart et al., Israel Math. Conf. Proc. 3, Weizmann, Jerusalem, 1990. MR 94e:11054 Zbl 0712.11029

[Kudla and Rallis 1994] S. S. Kudla and S. Rallis, "A regularized Siegel-Weil formula: the first term identity", Ann. of Math. (2) 140:1 (1994), 1-80. MR 95f:11036 Zbl 0818.11024

[Kudla and Sweet 1997] S. S. Kudla and W. J. Sweet, Jr., "Degenerate principal series representations for U(n, n)”, Israel J. Math. 98 (1997), 253-306. MR 98h:22021 Zbl 0896.22007

[Lapid and Rallis 2005] E. M. Lapid and S. Rallis, "On the local factors of representations of classical groups", pp. 309-359 in Automorphic representations, L-functions and applications: progress and prospects, edited by J. W. Cogdell et al., Ohio State Univ. Math. Res. Inst. Publ. 11, de Gruyter, Berlin, 2005. MR 2006j:11071 Zbl 1188.11023

[Lee 2007] S. T. Lee, "Covariants of $\operatorname{Sp}_{n}(\mathbb{C})$ and degenerate principal series of $\mathrm{GL}_{n}(\mathbb{W})$ ", J. Funct. Anal. 253:1 (2007), 18-42. MR 2008i:22010 Zbl 1139.22011

[Lee and Zhu 1998] S. T. Lee and C.-B. Zhu, "Degenerate principal series and local theta correspondence”, Trans. Amer. Math. Soc. 350:12 (1998), 5017-5046. MR 99c:22021 Zbl 0901.22008

[Li 1989] J.-S. Li, "Singular unitary representations of classical groups", Invent. Math. 97:2 (1989), 237-255. MR 90h:22021 Zbl 0694.22011

[Mínguez 2009] A. Mínguez, "Sur l'irréductibilité d'une induite parabolique", J. Reine Angew. Math. 629 (2009), 107-131. MR 2010h:22025 Zbl 1172.22008

[Mœglin et al. 1987] C. Mœglin, M.-F. Vignéras, and J.-L. Waldspurger, Correspondances de Howe sur un corps p-adique, Lecture Notes in Mathematics 1291, Springer, Berlin, 1987. MR 91f:11040 Zbl 0642.22002

[Piatetski-Shapiro and Rallis 1987a] I. Piatetski-Shapiro and S. Rallis, " $L$-functions for the classical groups", pp. 1-52 in Explicit constructions of automorphic L-functions, edited by S. Gelbart et al., Lecture Notes in Math. 1254, Springer, Berlin, 1987.

[Piatetski-Shapiro and Rallis 1987b] I. Piatetski-Shapiro and S. Rallis, "Rankin triple $L$ functions", Compos. Math. 64:1 (1987), 31-115. MR 89k:11037 Zbl 0637.10023

[Sahi 1995] S. Sahi, "Jordan algebras and degenerate principal series", J. Reine Angew. Math. 462 (1995), 1-18. MR 96d:22022 Zbl 0822.22006

[Scharlau 1985] W. Scharlau, Quadratic and Hermitian forms, Grundlehren der Mathematischen Wissenschaften [Fundamental Principles of Mathematical Sciences] 270, Springer, Berlin, 1985. MR 86k:11022 Zbl 0584.10010

[Sécherre 2009] V. Sécherre, "Proof of the Tadić conjecture (U0) on the unitary dual of $\mathrm{GL}_{m}(D)$ ", $J$. Reine Angew. Math. 626 (2009), 187-203. MR 2009k:22027 Zbl 1170.22009

[Tan 1998] V. Tan, "A regularized Siegel-Weil formula on U(2, 2) and U(3)", Duke Math. J. 94:2 (1998), 341-378. MR 99f:11065 Zbl 0989.11025

[Tan 1999] V. Tan, "Poles of Siegel Eisenstein series on U(n, n)", Canad. J. Math. 51:1 (1999), 164-175. MR 2000e:11073 Zbl 0963.11028

[Wallach 1988] N. R. Wallach, "Lie algebra cohomology and holomorphic continuation of generalized Jacquet integrals", pp. 123-151 in Representations of Lie groups (Kyoto, Hiroshima, 1986), edited by K. Okamoto and T. Oshima, Adv. Stud. Pure Math. 14, Academic Press, Boston, MA, 1988. MR 91d:22014 Zbl 0714.17016

[Weil 1965] A. Weil, "Sur la formule de Siegel dans la théorie des groupes classiques", Acta Math. 113 (1965), 1-87. MR 36 \#6421 Zbl 0161.02304 
[Weil 1974] A. Weil, Basic number theory, 3rd ed., Die Grundlehren der Mathematischen Wissenschaften 144, Springer, New York, 1974. MR 55 \#302 Zbl 0326.12001

[Yamana 2011] S. Yamana, "On the Siegel-Weil formula: the case of singular forms", Compos. Math. 147:4 (2011), 1003-1021. MR 2822859 Zbl 05938388

[Yamana 2013] S. Yamana, "On the Siegel-Weil formula for quaternionic unitary groups", Amer. J. Math (2013). To appear.

[Zhang 1995] G. K. Zhang, "Jordan algebras and generalized principal series representations", Math. Ann. 302:4 (1995), 773-786. MR 97h:22012 Zbl 0829.22023

Received March 12, 2012.

SHUNSUKE YAMANA

FACULTY OF MATHEMATICS

KYUSHU UNIVERSITY

744, NISHI-KU

MOTOOKA 819-0395

JAPAN

yamana@math.kyushu-u.ac.jp 


\title{
PACIFIC JOURNAL OF MATHEMATICS
}

\author{
msp.org/pjm
}

Founded in 1951 by E. F. Beckenbach (1906-1982) and F. Wolf (1904-1989)

\section{EDITORS}

V. S. Varadarajan (Managing Editor)

Department of Mathematics

University of California

Los Angeles, CA 90095-1555

pacific@math.ucla.edu

Paul Balmer

Department of Mathematics

University of California

Los Angeles, CA 90095-1555

balmer@math.ucla.edu

Daryl Cooper

Department of Mathematics

University of California

Santa Barbara, CA 93106-3080 cooper@math.ucsb.edu

Jiang-Hua $\mathrm{Lu}$

Department of Mathematics

The University of Hong Kong

Pokfulam Rd., Hong Kong jhlu@maths.hku.hk
Don Blasius

Department of Mathematics University of California

Los Angeles, CA 90095-1555

blasius@math.ucla.edu

Robert Finn

Department of Mathematics Stanford University

Stanford, CA 94305-2125

finn@math.stanford.edu

Sorin Popa

Department of Mathematics

University of California

Los Angeles, CA 90095-1555

popa@math.ucla.edu

Paul Yang

Department of Mathematics

Princeton University

Princeton NJ 08544-1000

yang@math.princeton.edu

\section{PRODUCTION}

Silvio Levy, Scientific Editor, production@msp.org

\section{SUPPORTING INSTITUTIONS}

ACADEMIA SINICA, TAIPEI

CALIFORNIA INST. OF TECHNOLOGY

INST. DE MATEMÁTICA PURA E APLICADA

KEIO UNIVERSITY

MATH. SCIENCES RESEARCH INSTITUTE

NEW MEXICO STATE UNIV.

OREGON STATE UNIV.

\author{
STANFORD UNIVERSITY \\ UNIV. OF BRITISH COLUMBIA \\ UNIV. OF CALIFORNIA, BERKELEY \\ UNIV. OF CALIFORNIA, DAVIS \\ UNIV. OF CALIFORNIA, LOS ANGELES \\ UNIV. OF CALIFORNIA, RIVERSIDE \\ UNIV. OF CALIFORNIA, SAN DIEGO \\ UNIV. OF CALIF., SANTA BARBARA
}

\author{
Vyjayanthi Chari \\ Department of Mathematics \\ University of California \\ Riverside, CA 92521-0135 \\ chari@math.ucr.edu \\ Kefeng Liu \\ Department of Mathematics \\ University of California \\ Los Angeles, CA 90095-1555 \\ liu@math.ucla.edu \\ Jie Qing \\ Department of Mathematics \\ University of California \\ Santa Cruz, CA 95064 \\ qing@cats.ucsc.edu
}

These supporting institutions contribute to the cost of publication of this Journal, but they are not owners or publishers and have no responsibility for its contents or policies.

See inside back cover or msp.org/pjm for submission instructions.

The subscription price for 2013 is US \$400/year for the electronic version, and \$485/year for print and electronic.

Subscriptions, requests for back issues and changes of subscribers address should be sent to Pacific Journal of Mathematics, P.O. Box 4163, Berkeley, CA 94704-0163, U.S.A. The Pacific Journal of Mathematics is indexed by Mathematical Reviews, Zentralblatt MATH, PASCAL CNRS Index, Referativnyi Zhurnal, Current Mathematical Publications and the Science Citation Index.

The Pacific Journal of Mathematics (ISSN 0030-8730) at the University of California, c/o Department of Mathematics, 798 Evans Hall \#3840, Berkeley, CA 94720-3840, is published monthly except July and August. Periodical rate postage paid at Berkeley, CA 94704, and additional mailing offices. POSTMASTER: send address changes to Pacific Journal of Mathematics, P.O. Box 4163, Berkeley, CA 94704-0163.

PJM peer review and production are managed by EditFLOW ${ }^{\circledR}$ from Mathematical Sciences Publishers.

PUBLISHED BY

mathematical sciences publishers

nonprofit scientific publishing

http://msp.org/

(C) 2013 Mathematical Sciences Publishers 


\section{PACIFIC JOURNAL OF MATHEMATICS}

Volume $264 \quad$ No. $1 \quad$ July 2013

On the center of fusion categories

ALAIN BRUGUIÈRES and ALEXIS VIRELIZIER

Connected quandles associated with pointed abelian groups

W. EdWin Clark, MoHamed ElHamdadi, Xiang-DONG HoU,

MASAHICO SAITO and TIMOTHY YEATMAN

Entropy and lowest eigenvalue on evolving manifolds

HongXin GuO, ROBERT PHILIPOWSKI and ANTON THALMAIER

Poles of certain residual Eisenstein series of classical groups

DIHUA JIANG, BAIYING LIU and LEI ZHANG

Harmonic maps on domains with piecewise Lipschitz continuous metrics 125

HAIGANG LI and CHANGYOU WANG

$q$-hypergeometric double sums as mock theta functions

JEREMY LOVEJOY and ROBERT OSBURN

Monic representations and Gorenstein-projective modules

XIU-HuA LUO and PU ZHANG

Helicoidal flat surfaces in hyperbolic 3-space

Antonio Martínez, JoÃo Paulo dos Santos and Keti

TENENBLAT

On a Galois connection between the subfield lattice and the multiplicative subgroup lattice

JOHN K. MCVEY

Some characterizations of Campanato spaces via commutators on Morrey 221 spaces

Shaoguang Shi and Shanzhen Lu

The Siegel-Weil formula for unitary groups 